\title{
Lung SBRT through Radiobiology
}

\author{
Aime M. Gloi \\ St. Vincent Hospital, Green Bay, USA \\ Email: agloi@sbcglobal.net
}

Received 4 January 2016; accepted 20 February 2016; published 23 February 2016

Copyright (C) 2016 by author and Scientific Research Publishing Inc.

This work is licensed under the Creative Commons Attribution International License (CC BY).

http://creativecommons.org/licenses/by/4.0/

(c) (7) Open Access

\section{Abstract}

Purpose: Stereotactic body radiation therapy (SBRT) has emerged as a standard treatment modality for medically inoperable early-stage lung cancer patients. The aim of this paper is to calculate radiobiological parameters for a sample of 39 patients who underwent lung SBRT. Materials and Methods: For SBRT, a typical regimen of 50 Gy in 4 - 5 fractions results in local tumor control rates around $99.9 \%$. We calculate dose volume histograms (DVHs) of targeted tumors and organs at risk for 39 patients. All patients received 4D imaging, and their internal treatment volumes (ITVs) were created by phase-based sorting of multiple CT datasets. Planning target volume (PTV) diameters ranged from 2.0 to $5.7 \mathrm{~cm}$. The DVHs for the PTV and organs at risk were analyzed using a Biosuite algorithm to calculate the equivalent uniform dose (EUD), tumor control probability (TCP) via a Poisson model, and normal tissue complication probability (NTCP) via an LKB model. The radiobiological effects were analyzed by correlating EUD and TCP with PTV volumes. Results: The mean PTV volume was $31.60 \pm 25.55 \mathrm{cc}$. The mean EUDs were $5.19 \pm 2.84,5.66 \pm 4.95,61.45 \pm$ $29.18,3.31 \pm 5.92,6.45 \pm 5.18$, and $12.22 \pm 5.94$ Gy for lungs, spinal cord, chest/ribs, heart, esophagus, and skin, respectively. On average, the heart had the lowest EUD and the chest/ribs had the highest $(61.45 \pm 29.18 \mathrm{~Gy})$. The mean NTCPs were estimated at $3.75 \% \pm 2.61 \%, 36.25 \% \pm$ $36.42 \%$, and $0.59 \% \pm 1.48 \%$, for the lungs, chest and esophagus, respectively. The NTCPs of spinal cord, heart, and skin were $0.00 \%$. The mean TCP value was $99.72 \% \pm 0.44 \%$. The mean BED value for our study was $109.49 \mathrm{~Gy}$. Conclusions: We have calculated radiobiological predictors based on DVHs for early-stage non-small cell lung cancer via SBRT. Our calculated predictors are compatible with previously published SBRT reports.

\section{Keywords}

BED, EUD, NSCLC, NTCP, TCP, SBRT

\section{Introduction}

Non-small cell lung cancer (NSCLC) continues to be the leading cause of cancer-related mortality [1]. Dose es- 
calation has been proposed to improve local control [2]-[5] and capitalize on the therapeutic ratio of thoracic radiotherapy, which is mainly limited by the potential toxicity to organs at risk (OARs). In brief, NSCLC is the most commonly diagnosed type of lung cancer that accounts for $85 \%$ of all cases characterized by slow growth and spread compared to other small cell lung cancer [6]. NSCLC encompasses a number of different types of lung cancer, which are categorized as "squamous" or "non-squamous". Smoking, ionizing radiation, occupational risks, environment, indoor air pollution and diseases as risk factors for lung cancer are the ultimate causes and risk factors. NSCLC is associated with persistent cough, shortness of breath, coughing up phlegm (sputum) with signs of blood, aches or pains when breathing or coughing, loss of appetite, fatigue, and loss of weight. NSCLC is detected using several options that include cytology, bronchoscopy, and needle biopsy. The Prognosis based on three year survival rates for lung cancer patient who received stereotactic body radiation therapy reveals a survival rate of $55.8 \%$ [7].

Stereotactic body radiation therapy (SBRT) is a technique that can attain a high cumulative biologically effective dose (BED) inside the tumor with a steep gradient outside the tumor, preventing dose to OARs. Achieving a high BED has been shown to improve patient survival and local control rates for many tumors [8]. However, SBRT confronts several issues including patient positioning, breathing patterns, and high dose escalation. All these issues can be detrimental to the treatment outcome.

Advancements in imaging and radiation treatment delivery systems have provided radiation oncologists with better target definition. For this reason, SBRT has recently emerged as a favored treatment modality for early-stage NSCLC. Modern SBRT has yielded durable local control results, comparable to surgery [9], and excellent overall survival outcomes even in inoperable patients.

Several authors have carried out studies on lung SBRT, examining the impacts of beam arrangement, image guidance, radiation dose constraints, dose prescription, and treatment planning algorithm on patient outcomes. For example, Papiez and Timmerman [10] discussed dose shaping and dose delivery techniques using standard beam arrangements with coplanar and noncoplanar beams. They established that SBRT treatment can be a new standard therapy for inoperable lung cancer. Chang and Timmerman [11] used SBRT along with recent advancements in diagnostic and real-time imaging to make precise measurements of tumor motion during treatment, and showed that the beams can be adjusted in real time to maintain the dose on the tumor. Through clinical trials of lung, liver, spine, pancreas, kidney, and prostate tumors, they proved that the effective doses delivered by SBRT are a comparable and noninvasive alternative to surgery.

However, much work remains to determine the best treatment parameters. Ding et al. [12] published a rationale with the Radiation Therapy Oncology Group (RTOG 0236) demonstrating the need for tissue heterogeneity corrections in the lung dose calculation. According to them, heterogeneity corrections have a major impact on the SBRT technique for lung tumors and normal lung tissues. This warning has been echoed by Herman et al. [13]. Another group directed by Timmerman et al. [14] uses tumor motion tracking methods such as breath-hold to achieve the lowest possible radiation dose to OARs. This group has suggested adopting a dose-response relationship for tumor control using SBRT, with increasing doses. Similarly, Xiao et al. [15] conducted a retrospective study to determine dose constraints and prescriptions for SBRT lung protocols and establish a heterogeneity correction for dose calculations. Likewise, Takeda et al. [16] performed a retrospective study of clinical outcomes in SBRT lung patients with Stages 1A and 1B NSCLC, and concluded that delivering a total of 50 Gy in 5 fractions at the periphery of the PTV is a viable treatment option. Finally, Takahashi et al. [17] compared the dose distributions calculated by three algorithms and revealed the importance of choosing an adequate algorithm for SBRT.

This research contributes to the literature by determining useful radiobiological parameters of SBRT treatments, such as the NTCP of organs at risk and the TCP of the tumor, in a sample of 39 patients. The results are calculated using BioSuite software, and will be compared to published data as quality assurance [18]-[20].

\section{Methods and Materials}

Ethical statement: Hospital Sisters Health System institutional review board approval was obtained for the treatment.

\subsection{Patients}

A cohort of thirty-nine patients with stage IA/IB NSCLC underwent definitive thoracic radiation as a single- 
mode treatment. All of them were treated by SBRT, but they were prescribed a variety of treatment plans. The demographics and stages of the patients are listed in Table 1. Volumetric modulated arc therapy (VMAT) and intensity modulated radiation therapy (IMRT) are the methods of choice for dose delivery; Figure 1 illustrates the VMAT method applied to one patient.

SBRT provided an adequate solution for challenging central and advanced lung tumors, because of the close proximity of organs at risk. VMAT dispenses the treatment more rapidly than IMRT, making the procedure

\begin{tabular}{cc} 
Table 1. Patient characteristics. \\
\hline Median age \\
Gender & 75.5 \\
Stage & 19 females vs. 20 males \\
Median tumor diameter & Stage III inoperable NSCLC \\
Median tumor volume & $3.7 \mathrm{~cm}$ \\
Location (upper/middle/lower) & $26.1 \mathrm{~cm}^{3}$ \\
Treatment modality & $13 / 17 / 9$ \\
\hline
\end{tabular}

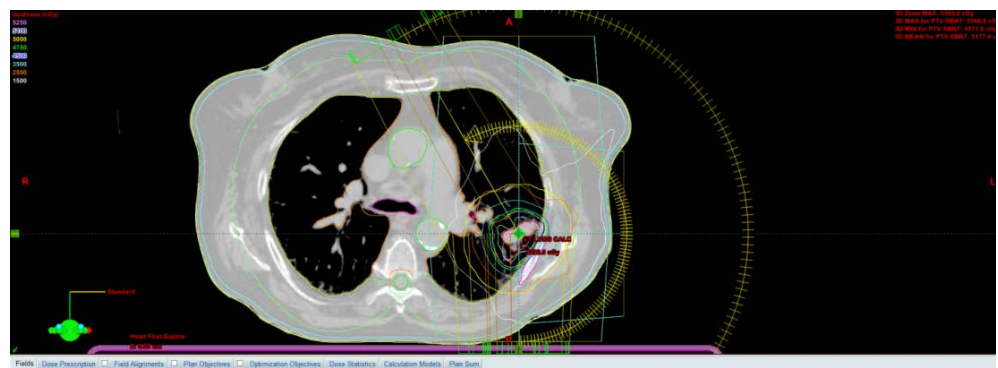

(a)

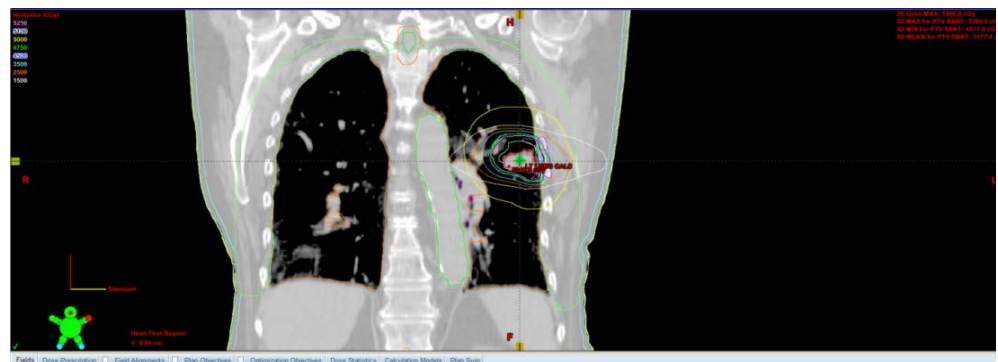

(b)

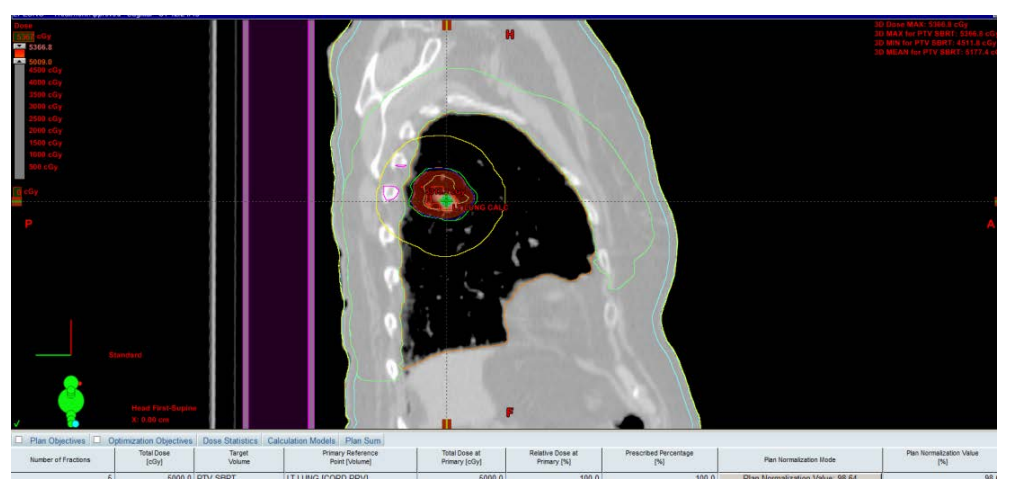

(c)

Figure 1. A 72-year-old woman with lung cancer was treated with SBRT for a total dose of 50 Gy in 5 fractions. (a) (top) VMAT with 3 arcs; (b) (middle) isodose lines; (c) (bottom) dose color “wash” normalized to $50 \mathrm{~Gy}$. 
more bearable for the patient. The specific advantages of its shorter duration are reduced intrafraction motion by the patient and less loss of biological effect.

\subsection{Dosimetry}

The patients were placed supine with their arms above their heads, while the tumor motion was assessed with 4DCT. The modalities of this procedure are described elsewhere [21]. Due to the treatment length for SBRT, close attention was paid to comfort as well as the reproducibility and stability of their position. Briefly, the computed tomography (CT) scans of all cases were acquired with $512 \times 512$ pixels and $0.25 \mathrm{~cm}$ slice spacing on the flat tabletop of a GE LightSpeed CT Scanner (Milwaukee, USA). The planning target volume (PTV) was created using a $5 \mathrm{~mm}$ wide isotropic expansion of the clinical target volume (CTV). The OARs, such as lungs, heart, chest, skin, esophagus and spinal cord, were delineated using axial CT images. All cases were planned using the VMAT (RapidArc) technique or IMRT technique, in 6X mode at $600 \mathrm{MU} / \mathrm{min}$ dose rate. The treatment plans were inversely optimized using the progressive resolution optimizer (PRO) in Eclipse TPS version 11 (Varian Medical Systems, Palo Alto, CA), such that at least 95\% of the PTV received the prescription dose of 50 Gy in 4 - 5 fractions. The optimized plans were calculated using an analytical anisotropic algorithm (AAA) with tissue heterogeneity correction. Each calculated plan was then normalized such that $100 \%$ of the prescribed dose covered $95 \%$ of the PTV. The calculation grid was set to $2.5 \mathrm{~mm}$ for all cases. The DVHs (Figure 2) from the approved plans of the thirty-nine patients, for both tumors and OARs, were imported into BioSuite [22] to calculate important radiobiological parameters.

\subsection{Radiobiology Parameters}

Several radiobiological parameters are used to describe causes and effects when tissue is subjected to radiation ionization. Among these are the equivalent uniform dose (EUD), normal tissue complication probability (NTCP), and tumor control probability (TCP). All three can be analyzed with variation of model analysis. These parameters and their modes of calculation have been described elsewhere [23] [24]. Briefly, Niemierko characterized EUD as the theoretical dose which would cause the same biological effect as the actual inhomogeneous dose, if the former were distributed uniformly throughout the volume of the structure of interest [25] [26]. According to Niemierko, EUD is given by

$$
E U D=\left(\sum_{i} v_{i} D_{i}^{a}\right)^{\frac{1}{a}}
$$

In this equation, $v_{i}$ is unitless and denotes the $i^{\text {th }}$ partial volume receiving dose $D_{i}$ in Gy. The value $a$ is a unitless model parameter specific to the OAR or tumor of interest. This formula has seen extensive use because of its simplicity. NTCP and TCP can be calculated using dose volume histograms (DVHs), combined

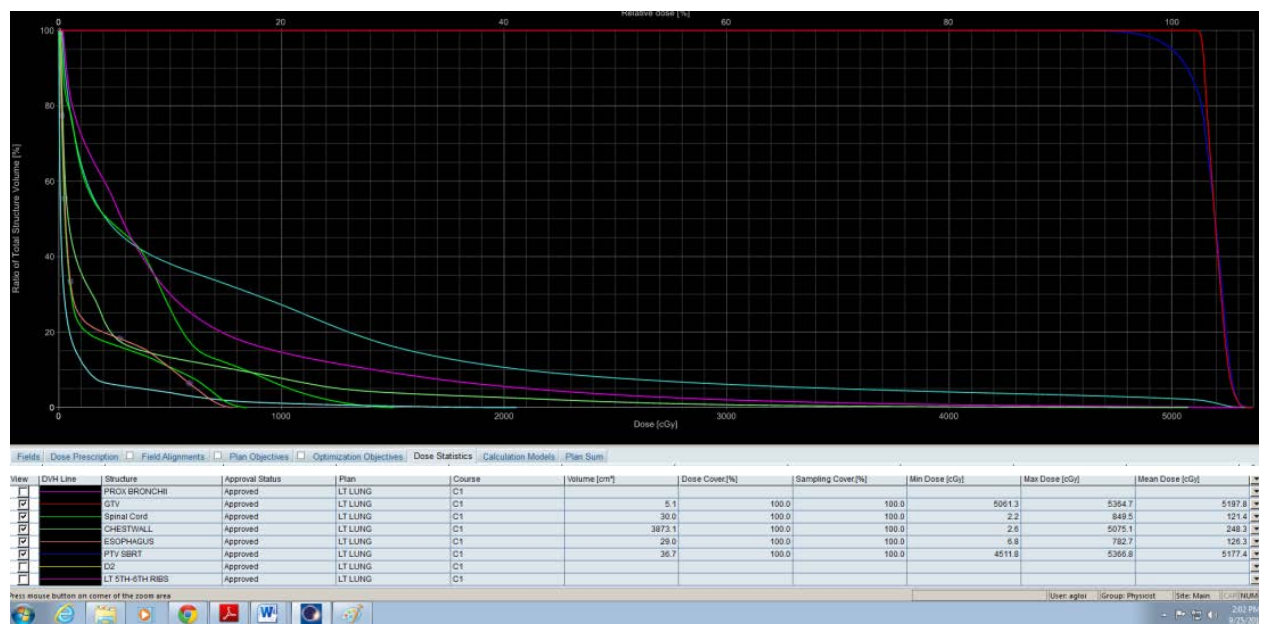

Figure 2. Dose volume histogram of lung SBRT treated at $50 \mathrm{~Gy}$ in 5 fractions. 
with additional parameters portraying the reactions of various tissues to ionizing radiation, including the $\alpha / \beta$ ratio, a parameter $n$ that controls dose volume effects, $\mathrm{TD}_{50}$ or dose sensitivity, a parameter $m$ that controls the slope of dose response curve, and the reference volume $v$ [27]-[30]. This study uses Biosuite software to model NTCP and TCP, based in part on inputs from the Lyman-Kutcher-Burman (LKB) model parameters. NTCP was calculated for OARs and linear quadratic (LQ) Poisson "Marsden” model [31], to estimate TCP for lung tumors.

\section{Results}

Data from thirty-nine patients who underwent SBRT lung treatment were used for this study (Figure 3(a), Figure 3(b)).

Their prescribed doses were either 5 daily fractions of 10 Gy or 4 daily fractions of $12.5 \mathrm{~Gy}$, for a total dose of 50 Gy using SBRT. The patient characteristics are summarized in Table 1.

The primary organs involved were the lung, heart, esophagus, spinal cords, chest wall, and skin. The median tumor size was $3.7 \mathrm{~cm}$ (range 1.6 to $6.0 \mathrm{~cm}$ ). Eleven patients $(28.20 \%$ ) had a tumor size of $<3 \mathrm{~cm}$, and 28 patients (71.80\%) had a tumor size of $\geq 3 \mathrm{~cm}$. The tumors were located in different areas of the lung: upper (13), middle (17), or lower (9). Among the 39 patients, four received high biologically effective doses of radiation (BED > 151.2 Gy), 11 received medium-high doses (BED, 112.5 Gy), five received medium-low doses (105.6 Gy), and 19 received low doses (BED = $100 \mathrm{~Gy}$ ). Table 2 shows the characteristics of the four BED groups.

According to the LQ model, the median BED is 100 and the range is $100-151.2 \mathrm{~Gy}$. The mean PTV volume is $31.60 \pm 25.55 \mathrm{cc}$. The mean EUDs in Gy to the different organs at risk are $5.19 \pm 2.84$ (lungs), $5.66 \pm 4.95$ (spinal cord), $61.45 \pm 29.18$ (chest wall), $3.31 \pm 5.92$ (heart), $6.45 \pm 5.18$ (esophagus), and $12.22 \pm 5.94$ (skin). On average, the heart received the lowest EUD, while the chest and ribs received the highest (61.45 $\pm 29.18 \mathrm{~Gy}$ ). Figure 4 summarizes all observed EUDs for the six OARs and thirty-nine cases.

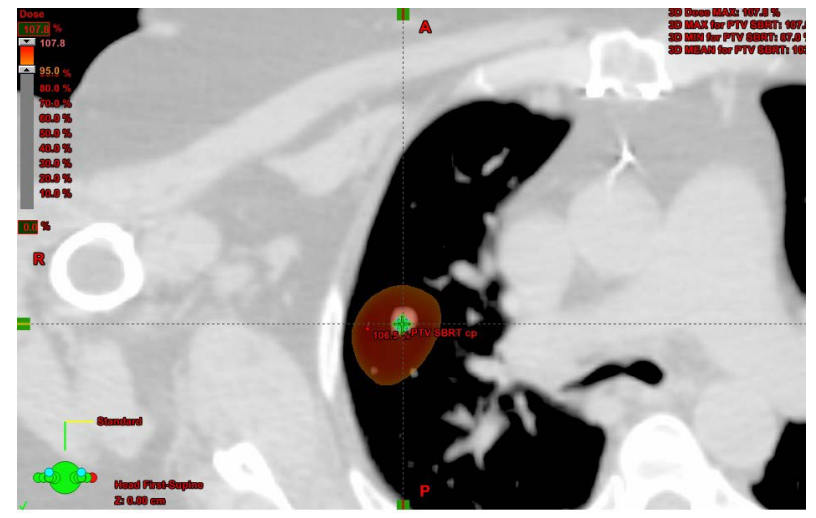

(a)

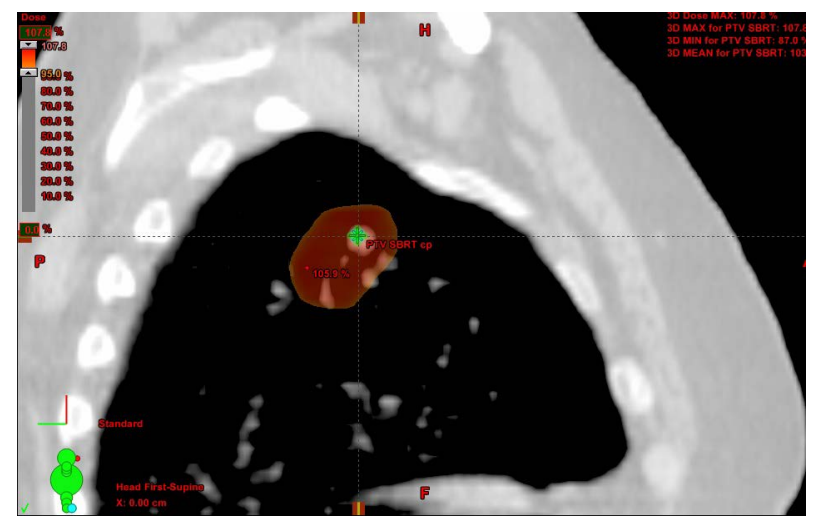

(b)

Figure 3. A 70-year-old woman with lung cancer was treated with SBRT for a total dose of 50 Gy given in 5 fractions. 
Table 2. Tumor size by BED group.

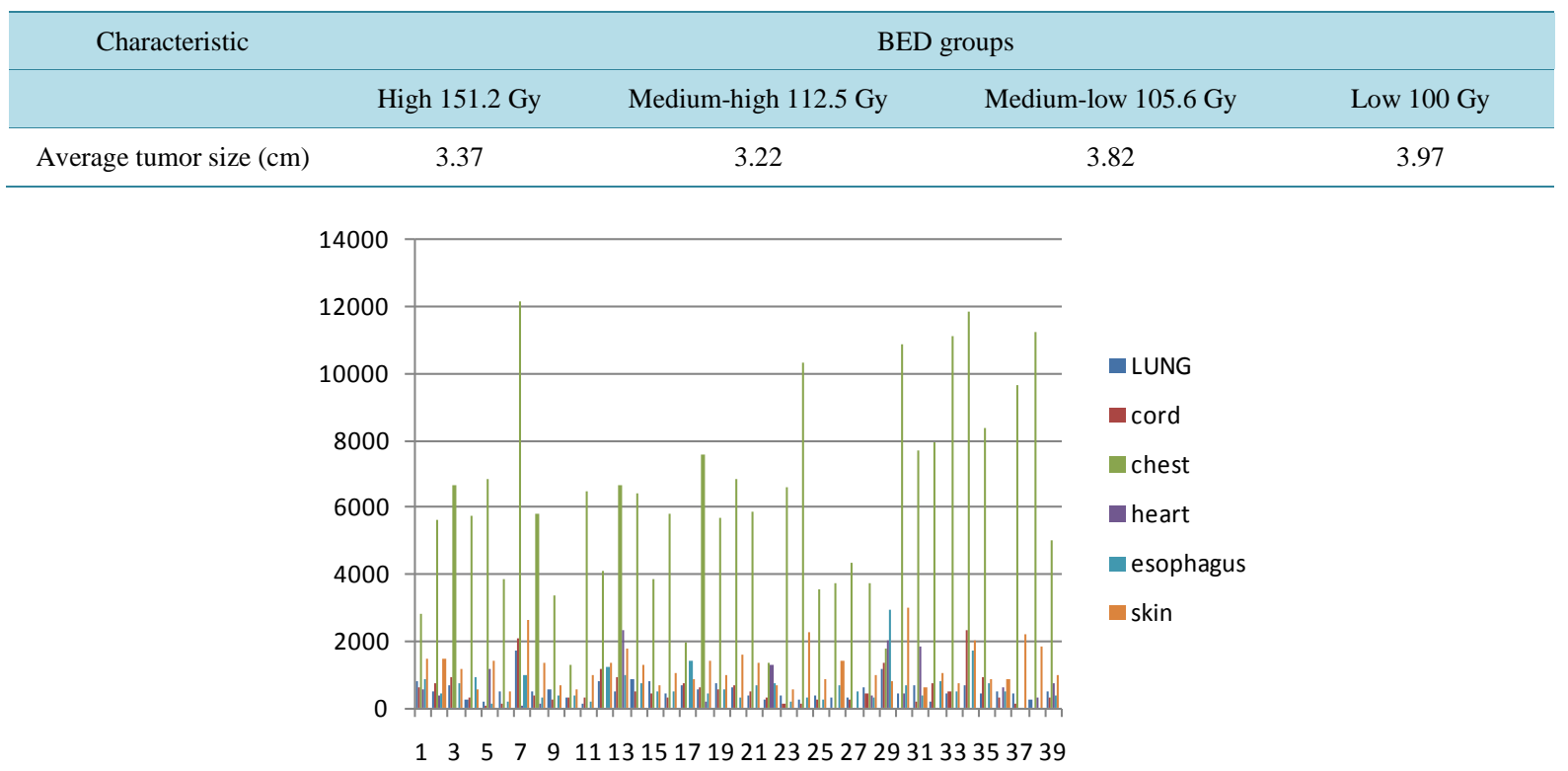

Figure 4. EUDs for organs at risk in lung SBRT. (The y-axis is in cGy and the $\mathrm{x}$-axis is the patient number).

The mean NTCPs were $3.75 \% \pm 2.61 \%, 36.25 \% \pm 36.42 \%$, and $0.59 \% \pm 1.48 \%$ for the lungs, chest, and esophagus, respectively. The NTCP values for cords, heart, and skin were $0.00 \%$. All NTCP values are shown in Figure 5.

The mean TCP was $99.72 \% \pm 0.44 \%$. Using the PTV encompassing dose or maximum (isocentric) dose, our data indicate that the mean isocenter dose is $53.08 \pm 2.41 \mathrm{~Gy}$. Figure 6 displays the BED value at the isocenter, the isocenter dose, and the TCP for all patients.

\section{Discussion}

This research employs radiobiological modeling to calculate the biological effects of radiation therapy based on the linear-quadratic (LQ) model. In this study, we analyzed radiobiological parameters for thirty-nine patients who underwent lung SBRT. Several predictive factors such as TCP, BED, EUD, and NTCP were characterized using the LKB model. All input parameters of this study are derived from the reports of Burman et al. [32] and Luxton et al. [33].

For patients who undergo lung SBRT, the most obvious toxicities are pneumonitis, esophagitis, and chestwall pain; pulmonary and cardiovascular complications are also very common. Accordingly, within our sample the NTCP values for the cords, heart, and skin are $0.00 \%$. The highest observed NTCP value is for the chest, while smaller but still significant NTCP values are observed for the total lung, treated lung, and esophagus. The large value of NTCP for the chest can be attributed to several factors, including tumor location and some issues that are patient-dependent. For example, it is believed that female gender and a small tumor-chest wall separation are both risk factors for rib fracture [34].

Uematsu et al. [35] described a 5-year experience with 50 patients who underwent SBRT, using 50 - 60 Gy in 5 - 10 fractions with a median follow-up of 36 months. In their sample only one patient (2\%) had a rib fracture detected by follow-up CT, and this one did not require medical treatment. Uematsu's study is supported by the work of Nambu et al. [36], who states that the frequency and degree of chest wall pain is greatly dependent on the total dose or fraction. They suggested that a $\mathrm{BED}_{10}$ ranging from 96 to $119 \mathrm{~Gy}$ at the isocenter is safe enough to prevent any chest wall adverse reaction. However, different frequencies are expected at the horizons as we follow-up our patients.

We used the LQM model with $\alpha / \beta=10$ Gy for our BED calculations and compared the results to published reports with a variety of SBRT schemes. In addition, we examine the dose-response relationship for local tumor 


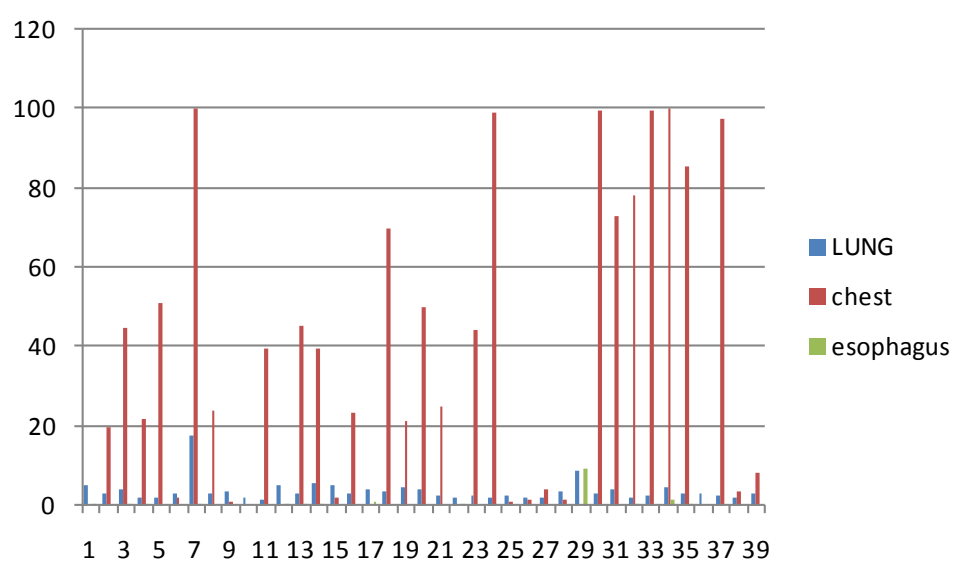

Figure 5. NTCPs for organs at risk in lung SBRT (The y-axis is a \% probability, and the $\mathrm{x}$-axis is the patient number).

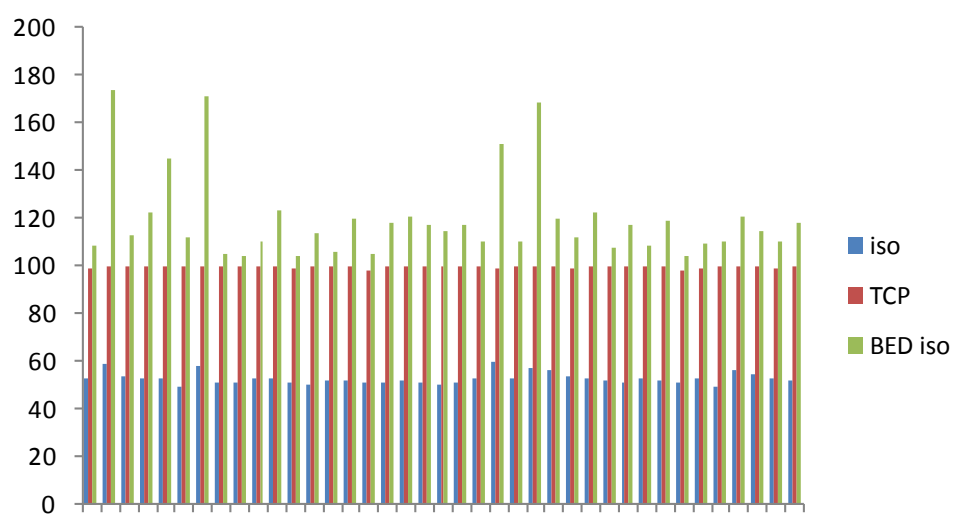

$1 \quad 3 \quad 5 \quad 7 \quad 9111315171921232527293133353739$

Figure 6. TCP, BED at the isocenter, and isocenter dose for lung SBRT The $y$-axis is in Gy and the $\mathrm{x}$-axis represents the patient number).

control. To achieve better local tumor control, the consensus in the literature is that the BED should be over 100 Gy. The mean BED value for our study, estimated at the isocenter, was $119.57 \mathrm{~Gy}$. This is consistent with reviews by Onishi et al. [37] and Baumann et al. [38] which state that higher BED values result in better outcomes. They showed, considering a variety of treatment schemes, that BED $\geq 100 \mathrm{~Gy}$ results in a recurrence rate of only $8.4 \%$, while patients receiving BED $<100$ Gy have a recurrence rate of $26.4 \%$.

According to Zhang et al. [39], BED > $146 \mathrm{~Gy}$ resulted in a local control rate of $100 \%$, two years after treatment. The BEDs in our study range from 100 - 151.2 Gy. It is believed that in order for a lower BED to maximize tumor control, it is paramount to avoid complications and effectively treat tumors in central locations.

In this study, the SBRT treatments were performed with either IMRT or VMAT. Both techniques are susceptible to interplay between the multileaf collimator (MLC) and tumor respiratory motion. Li et al. [40] evaluated the interplay effect for intra-fraction cumulative dose and concluded that less than $1 \%$ discrepancy in the PTV and ITV minimum doses was observed using an energy mapping algorithm. However, in a SBRT scenario, where 3 - 5 dose fractions are delivered, the effect of this interplay on dose distributions is not well defined.

Examining the TCP and NTCP results, dose calculation with an analytical anisotropic algorithm (AAA) may become more and more clinically significant for lung cancer. It is paramount to use the most accurate dose calculation algorithm possible, in order to avoid underestimation of the required dose and increasing the probability of local disease recurrence. On the other hand, overestimation of the dose will increase toxicity to normal tissues. For example, Latifi et al. [41] show a higher recurrence rate for patients whose treatments were planned with Pencil-Beam (PB) compared to collapsed cone convolution (CCC) algorithms, even though the prescribed nominal dose and constraints were identical. 
This study has several limitations, including the lack of patient follow-up in order to establish a survival curve. Only randomized assessments among patients undergoing the SBRT regimen will avoid the disparities and selection biases present in these comparisons.

Another limitation is that LQM was used to calculate radiobiological parameters. Timmerman et al. [42] [43] showed that for SBRT with a dose greater than $50 \mathrm{~Gy}$, cell survival curves seem linear rather than downward bending, as LQM would suggest. For this reason, we believe that LQM may overestimate the cytotoxic effects of SBRT. A new method called the universal survival curve (USC) model has been introduced which associates the LQM for the low dose region with a multitarget model asymptote for high dose region [44]. In addition, inconsistencies on endpoints due to variation in breathing patterns of the lung, making more difficult to rely solely on DVH-based parameters. EUD, NTCP, and TCP can be calculated directly from the DVH. These parameters are therefore dependent on of the treatment planning system (TPS) employed, which includes interpolation, binning, and normalization methods. The quantitative evaluation of EUD, NTCP, and TCP reveals a strong dependence on the actual clinical treatment situation. Finally, SBRT radiation dose and fractionation depends on the oncologist's experience and also varies due to a lack of consensus in the literature regarding recommended BED. In addition, tailored treatment planning has to account for organ motion and real-time tomographic acquisition.

\section{Conclusion}

NTCP, EUD, and TCP may serve as a useful tool for prospective and retrospective analysis of radiotherapy treatment plans in lung SBRT.

\section{References}

[1] Riess, J.W. and Wakelee, H.A. (2012) Metastatic Non-Small Cell Lung Cancer Management: Novel Targets and Recent Clinical Advances. Clinical Advances in Hematology \& Oncology, 10, 226-234.

[2] Kong, F.M., Ten Haken, R.K., Schipper, M.J., Sullivan, M.A., Chen, M., Lopez, C., et al. (2005) High-Dose Radiation Improved Local Control and Overall Survival in Patients with Inoperable/Unresectable Non-Small-Cell Lung Cancer: Long-Term Results of a Radiation Dose Escalation Study. International Journal of Radiation Oncology * Biology * Physics, 63, 324-333. http://dx.doi.org/10.1016/j.ijrobp.2005.02.010

[3] Rosenzweig, K.E., Fox, J.L., Yorke, E., Amols, H., Jackson, A., Rusch, V., et al. (2005) Results of a Phase I DoseEscalation Study Using Three-Dimensional Conformal Radiotherapy in the Treatment of Inoperable Nonsmall Cell Lung Carcinoma. Cancer, 103, 2118-2127. http://dx.doi.org/10.1002/cncr.21007

[4] Willner, J., Baier, K., Caragiani, E., Tschammler, A. and Flentje, M. (2002) Dose, Volume, and Tumor Control Predictions in Primary Radiotherapy of Non-Small-Cell Lung Cancer. International Journal of Radiation Oncology * Biology * Physics, 52, 382-389. http://dx.doi.org/10.1016/S0360-3016(01)01823-5

[5] Bradley, J., Graham, M.V., Winter, K., Purdy, J.A., Komaki, R. and Roa, W.H. (2005) Toxicity and Outcome Results of RTOG 9311: A Phase I-II Dose-Escalation Study Using Three-Dimensional Conformal Radiotherapy in Patients with Inoperable Non-Small-Cell Lung Carcinoma. International Journal of Radiation Oncology * Biology * Physics, 61, 318-328. http://dx.doi.org/10.1016/j.ijrobp.2004.06.260

[6] Timmerman, R., Paulus, R., Galvin, J., Michalski, J., Straube, W., Bradley, J., Fakiris, A., Bezjak, A., Videtic, G., Johnstone, D., Fowler, J., Gore, E. and Choy, H. (2010) Stereotactic Body Radiation Therapy for Inoperable Early Stage Lung Cancer. JAMA, 303, 1070-1076. http://dx.doi.org/10.1001/jama.2010.261

[7] Barzi, A. and Pennell, N.A. (2010) Targeting Angiogenesis in Non-Small Cell Lung Cancer: Agents in Practice and Clinical Development. EJCMO, 2, 31-42.

[8] Feng, F.K., Zhao, J., Wang, J. and Faivre, F.C. (2014) Radiation Dose Effect in Locally Advanced Non-Small Cell Lung Cancer. Journal of Thoracic Disease, 6, 336-347.

[9] Mehta, N., King, C.R., Agazaryan, N., Steinberg, M., Hua, A. and Lee, P. (2012) Stereotactic Body Radiation Therapy and 3-Dimensional Conformal Radiotherapy for Stage I Non-Small Cell Lung Cancer: A Pooled Analysis of Biological Equivalent Dose and Local Control. Practical Radiation Oncology, 2, 288-295. http://dx.doi.org/10.1016/j.prro.2011.10.004

[10] Papiez, L. and Timmerman, R. (2008) Hypofractionation in Radiation Therapy and Its Impact. Journal of Medical Physics, 35, 112-118. http://dx.doi.org/10.1118/1.2816228

[11] Chang, B.K. and Timmerman, R.D. (2007) Stereotactic Body Radiation Therapy: A Comprehensive Review. Journal of Clinical Oncology, 30, 637-644. http://dx.doi.org/10.1097/COC.0b013e3180ca7cb1 
[12] Ding, M., Kavanagh, B.D. and Schefter, T. (2005) The Influence of Heterogeneity on Tumor and Normal Lung Dosimetry in Stereotactic Body Radiation Therapy for Lung Cancer Treatment. International Journal of Radiation Oncology * Biology * Physics, 63, S511-S512. http://dx.doi.org/10.1016/j.ijrobp.2005.07.867

[13] Herman, T.L., Gabrish, H. and Herman, T.S. (2010) Impact of Tissue Heterogeneity Corrections in Stereotactic Body Radiation Therapy Treatment Plans for Lung Cancer. Journal of Medical Physics, 35, 170-173. http://dx.doi.org/10.4103/0971-6203.62133

[14] Timmerman, R.D., Park, C. and Kavanagh, B.C. (2007) The North American Experience with Stereotactic Body Radiation Therapy in Non-Small Cell Lung Cancer. Journal of Thoracic Oncology, 2, 101-112.

[15] Xiao, Y., Papiez, L. and Paulus, R. (2009) Dosimetric Evaluation of Heterogeneity Corrections for RTOG 0236: Stereotactic Body Radiotherapy of Inoperable Stage I-II Non-Small Cell Lung Cancer. International Journal of Radiation Oncology * Biology * Physics, 73, 1235-1242. http://dx.doi.org/10.1016/j.ijrobp.2008.11.019

[16] Takeda, A., Sanuki, N. and Kunieda, E. (2009) Stereotactic Body Radiotherapy for Primary Lung Cancer at a Dose of 50 Gy Total in Five Fractions to the Periphery of the Planning Target Volume Calculated Using a Superposition Algorithm. International Journal of Radiation Oncology *Biology* Physics, 73, 442-448. http://dx.doi.org/10.1016/j.ijrobp.2008.04.043

[17] Takahashi, W., Yamashita, H. and Saotome, N. (2012) Evaluation of Heterogeneity Dose Distributions for Stereotactic Radiotherapy (SRT): Comparison of Commercially Available Monte Carlo Dose Calculation with Other Algorithms. Journal of Radiation Oncology, 7, 20. http://dx.doi.org/10.1186/1748-717X-7-20

[18] Onishi, H., Nagata, Y., Shirato, H., Arimoto, T., Gomi, K., Karasawa, K., Hayakawa, K., Takai, Y., Ouchi, A. and Takeda, A. (2007) Stereotactic Body Radiotherapy (SBRT, BED $\geq 100$ Gy) for Operable Stage I Non-Small Cell Lung Cancer: Is SBRT Comparable to Surgery? International Journal of Radiation Oncology *Biology* Physics, 69, S86S87.

[19] Park, S., Urm, S. and Cho, H. (2014) Analysis of Biologically Equivalent Dose of Stereotactic Body Radiotherapy for Primary and Metastatic Lung Tumors. Cancer Research and Treatment, 46, 403-410. http://dx.doi.org/10.4143/crt.2013.168

[20] Kong, F.-M., Zhao, J. and Wang, J.B. (2014) Corrine Faivre-Finn: Radiation Dose Effect in Locally Advanced NonSmall Cell Lung Cancer. Journal of Thoracic Disease, 6, 336-347.

[21] Glide-Hurst, C.K., Smith, M.S., Ajlouni, M. and Chetty, I.J. (2013) Evaluation of Two Synchronized External Surrogates for 4D CT. Journal of Applied Clinical Medical Physics, 14, 4301.

[22] Uzan, J. and Nahum, A.E. (2012) Radiobiologically Guided Optimization of the Prescription Dose and Fractionation Scheme in Radiotherapy Using BioSuite. The British Journal of Radiology, 85, 1279-1286. http://dx.doi.org/10.1259/bjr/20476567

[23] Niemierko, A. (1997) Reporting and Analyzing Dose Distributions: A Concept of Equivalent Uniform Dose. Medical Physics, 24, 103-110. http://dx.doi.org/10.1118/1.598063

[24] Gay, H.A. and Niemierko, A. (2007) A Free Program for Calculating EUD-Based NTCP and TCP in External Beam Radiotherapy. Medical Physics, 23, 115-125. http://dx.doi.org/10.1016/j.ejmp.2007.07.001

[25] Lyman, J.T. (1985) Complication Probability as Assessed from Dose-Volume Histograms. Radiation Research, 8, S13S19. http://dx.doi.org/10.2307/3583506

[26] Lyman, J.T. and Wolbarst, A.B. (1987) Optimization of Radiation Therapy. III. A Method of Assessing Complication Probabilities from Dose-Volume Histograms. International Journal of Radiation Oncology *Biology* Physics, 13, 103-109. http://dx.doi.org/10.1016/0360-3016(87)90266-5

[27] Kutcher, G.J. and Burman, C. (1989) Calculation of Complication Probability Factors for Non-Uniform Normal Tissue Irradiation: The Effective Volume Method. International Journal of Radiation Oncology *Biology* Physics, 16, 16231630. http://dx.doi.org/10.1016/0360-3016(89)90972-3

[28] Wheldon, T.E., Deehan, C., Wheldon, E.G. and Barrett, A. (1998) The Linear-Quadratic Transformation of Dose-Volume Histograms in Fractionated Radiotherapy. Radiotherapy and Oncology, 46, 285-295. http://dx.doi.org/10.1016/S0167-8140(97)00162-X

[29] Niemierko, A. (1999) A Generalized Concept of Equivalent Uniform Dose (EUD). Medical Physics, 26, 1100.

[30] Luxton, G., Keall, P.J. and King, C.R. (2008) A New Formula for Normal Tissue Complication Probability (NTCP) as a Function of Equivalent Uniform Dose (EUD). Physics in Medicine and Biology, 53, 23-36. http://dx.doi.org/10.1088/0031-9155/53/1/002

[31] Nahum, A.E. and Sanchez-Nieto, B. (2001) Tumor Control Probability Modelling: Basic Principles and Applications in Treatment Planning. Physica Medica, 17, 13-23.

[32] Burman, C., Kutcher, G.J., Emami, B. and Goitein, M. (1991) Fitting of Normal Tissue Tolerance Data to an Analytic 
Function. International Journal of Radiation Oncology *Biology* Physics, 21, 123-135. http://dx.doi.org/10.1016/0360-3016(91)90172-Z

[33] Luxton, G., Keall, P.J. and King, C.R. (2008) A New Formula for Normal Tissue Complication Probability (NTCP) as a Function of Equivalent Uniform Dose (EUD). Physics in Medicine and Biology, 53, 23-36. http://dx.doi.org/10.1088/0031-9155/53/1/002

[34] Kim, S.S., Song, S.Y., Kwak, J., Ahn, S.D., Kim, J.H. and Lee, J.S. (2013) Clinical Prognostic Factors and Grading System for Rib Fracture Following Stereotactic Body Radiation Therapy (SBRT) in Patients with Peripheral Lung Tumors. Lung Cancer, 79, 161-166. http://dx.doi.org/10.1016/j.lungcan.2012.10.011

[35] Uematsu, M., Shioda, A., Suda, A., Fukui, T., Ozeki, Y., Hama, Y., et al. (2001) Computed Tomography-Guided Frameless Stereotactic Radiotherapy for Stage I Nonsmall-Cell Lung Cancer: A 5-Year Experience. International Journal of Radiation Oncology *Biology* Physics, 51, 666-670. http://dx.doi.org/10.1016/S0360-3016(01)01703-5

[36] Nambu, A., Onishi, H., Aoki, S., Tominaga, L., Kuriyama, K., Araya, M., Saito, R., Maehata, Y., Komiyama, T., Marino, K., Koshiishi, T., Sawada, E. and Araki, T. (2013) Rib Fracture after Stereotactic Radiotherapy for Primary Lung Cancer: Prevalence, Degree of Clinical Symptoms, and Risk Factors. BMC Cancer, 13, 68. http://dx.doi.org/10.1186/1471-2407-13-68

[37] Onishi, H., Shirato, H., Nagata, Y., Hiraoka, M., Fujino, M. and Gomi, K. (2007) Hypofractionated Stereotactic Radiotherapy (Hypo-FXSRT) for Stage I Non-Small Cell Lung Cancer: Updated Results of 257 Patients in a Japanese Multi-Institutional Study. Journal of Thoracic Oncology, 2, S94-S100. http://dx.doi.org/10.1097/JTO.0b013e318074de34

[38] Baumann, P., Nyman, J., Lax, I., Friesland, S., Hoyer, M. and Rehn, E.S. (2006) Factors Important for Efficacy of Stereotactic Body Radiotherapy of Medically Inoperable Stage I Lung Cancer: A Retrospective Analysis of Patients Treated in the Nordic Countries. Acta Oncologica, 45, 787-795. http://dx.doi.org/10.1080/02841860600904862

[39] Zhang, J., Yang, F., Li, B., Li, H., Liu, J., Huang, W., et al. (2011) Which Is the Optimal Biologically Effective Dose of Stereotactic Body Radiotherapy for Stage I Non-Small-Cell Lung Cancer? A Meta-Analysis. International Journal of Radiation Oncology *Biology* Physics, 81, e305-e316. http://dx.doi.org/10.1016/j.jjrobp.2011.04.034

[40] Li, H., Zhong, H., Kim, J., et al. (2011) Investigation of the Interplay Effect between MLC and Lung Tumor Motions Using 4DCT and RPM Profile Data. Medical Physics, 38, 3692-3693. http://dx.doi.org/10.1118/1.3612838

[41] Latifi, K., Oliver, J., Baker, R., et al. (2014) Study of 201 Non-Small Cell Lung Cancer Patients Given Stereotactic Ablative Radiation Therapy Shows Local Control Dependence on Dose Calculation Algorithm. International Journal of Radiation Oncology *Biology* Physics, 88, 1108-1113. http://dx.doi.org/10.1016/j.ijrobp.2013.12.047

[42] Timmerman, R., McGarry, R., Yiannoutsos, C., et al. (2006) Excessive Toxicity When Treating Central Tumors in a Phase II Study of Stereotactic Body Radiation Therapy for Medically Inoperable Early Stage Lung Cancer. Journal of Clinical Oncology, 24, 4833-4839. http://dx.doi.org/10.1200/JCO.2006.07.5937

[43] Timmerman, R.D., Kavanagh, B.D., Cho, L.C., Papiez, L. and Xing, L. (2007) Stereotactic Body Radiation Therapy in Multiple Organ Sites. Journal of Clinical Oncology, 25, 947-952. http://dx.doi.org/10.1200/JCO.2006.09.7469

[44] Park, C., Papiez, L., Zhang, S., Story, M. and Timmerman, R.D. (2008) Universal Survival Curve and Single Fraction Equivalent Dose: Useful Tools in Understanding Potency of Ablative Radiotherapy. International Journal of Radiation Oncology *Biology* Physics, 70, 847-852. http://dx.doi.org/10.1016/j.ijrobp.2007.10.059 